\title{
External Morphology of Sensory Structures of Fourth Instar Larvae of Neotropical Species of Phlebotomine Sand Flies (Diptera: Psychodidae) under Scanning Electron Microscopy
}

\author{
Felipe Arley Costa Pessoa/ ${ }^{+}$, Raul Guerra de Queiroz, Richard Douglas Ward* \\ Coordenação de Pesquisas em Ciências da Saúde, Instituto Nacional de Pesquisas da Amazônia, Caixa Postal 478, \\ 69011-970 Manaus, AM, Brasil *School of Life Sciences, Keele University, Staffordshire, UK
}

\begin{abstract}
In the present study, some morphological structures of antennae, maxillary palps and caudal setae of fourth instar larvae of laboratory-reared phlebotomine sand flies (Lutzomyia longipalpis, L. migonei, L. evandroi, L. lenti, L. sericea, L. whitmani and L. intermedia) of the State of Ceará, Brazil, were examined under scanning electron microscopy. The antennal structures exhibited considerable variation in the morphology and position. A prominent digitiform distal segment has been observed only on the antenna of species of the subgenus Nyssomyia. The taxonomic relevance of this and other antennal structure is discussed. The papiliform structures found in the maxillae and the porous structures of the caudal setae of all species examined may have chemosensory function. Further studies with transmission electron microscopy are needed to better understand the physiological function of these external structures.
\end{abstract}

Key words: phlebotomine sand fly larvae - sensory structures - scanning electron microscopy

Phlebotomine sand flies (Diptera: Psychodidae) are insects of medical and veterinary importance, as they can transmit leishmaniases, bartonelloses and arboviruses. As with many insect groups, the morphological characters used to distinguish sand fly species are those of the adults, and the immature stages of most species have not been seen or described. Of about 400 species described in the Neotropics (Young \& Duncan 1994), only 66 species had their immatures studied (Ward 1976a, b).

The morphology of sand fly larvae was described in details by Perfil'ev (1968), Abonnenc (1972) and Forattini (1973). Up to that time, all descriptions and morphological observations were made by light microscopy. Studies of sand fly larvae with scanning electronic microscopy (SEM) were initiated by Killick-Kendrick et al. (1989) on Old World species Phlebotomus tobbi. In South America, Leite and Willians $(1996,1997)$ described by SEM the larvae of fourth and first instar of $L$. longipalpis. Fausto et al. (1998) and Pessoa et al.

\footnotetext{
This work was developed while RGQ was a WHO RSG/ TDR grantee (no. M8/181/Q.21).

${ }^{+}$Corresponding author. Fax +55-92-642.3061. E-mail: pessoa@inpa.gov.br

Received 15 February 2001

Accepted 30 July 2001
}

(2000) studied spiracles of South American sand fly larvae.

Few studies on sensillae of immatures of dipteran vectors have been developed. Zacharuck et al. (1971), Zacharuck and Blue (1971) made some observations on antennae of larvae of Aedes aegypti with transmission electron microscopy (TEM). Lopes and Leite (1987) found probable structures in the integument of larvae of Sarcophagidae. Leite and Williams $(1996,1997)$ mentioned probable sensillae on the integument, antennae and mouth structures of L. longipalpis. Coscarón et al. (1998) also observed sensory structures in larvae of Tabanidae with SEM.

In the present study, some morphological structures of antennae, papillae of maxillary palps and caudal setae of fourth instar larvae of Neotropical phlebotomine sandflies were studied by SEM.

\section{MATERIALS AND METHODS}

Fourth instar larvae of the following species from the State of Ceará, Brazil, were considered in this study: L. longipalpis (Sobral, $3^{\circ} 45^{\prime} \mathrm{S}, 40^{\circ} 5^{\prime} \mathrm{W}$ ), L. migonei, L. evandroi, L. lenti, L. sericea and $L$. whitmani (Baturité, $4^{\circ} 20^{\prime} \mathrm{S}, 38^{\circ} 55^{\prime} \mathrm{W}$ ), and $L$. intermedia (Crato, 7014'03'"S, 39 24'34'W). The specimens were obtained from laboratory colonies, established and maintained according to the methods by Killick-Kendrick and Killick-Kendrick (1991). The larvae were killed in hot water $\left(70^{\circ} \mathrm{C}\right)$, fixed in $3 \%$ glutaraldehyde and then washed thoroughly in 
phosphate-buffered saline, the solution being changed every 30 min during $6 \mathrm{~h}$. They were then fixed in osmium tetroxide, dehydrated in a series of ethyl alcohol concentrations, submitted to critical point drying in carbon dioxide and spattered with 25 MA colloidal gold. Three specimens of each species were examined with a Hitachi S 4500 SEM.

\section{RESULTS}

\section{Antennae}

L. longipalpis - Each antenna (Fig. 1) has a basal tubercle (socket), a small and cylindrical segment fused at a second ovoid distal segment. This segment presents an antennal organ equipped with a longitudinal furrow in the posterior surface and three short structures in the base of the segment, and a long sensillae with several pores (multiporous sensillae) (Fig. 2), that begins in the base of the first segment. This porous structure is inserted in the furrow. There is a small digitiform appendix, in the centre of the apex of the distal segment, also porous.

L. whitmani and L. intermedia - Each antenna of both species bears a tubercle with the shape of a truncate cone, a short basal segment, and a prominent digitiform distal segment (Figs 3,4). As in the antenna of L. longipalpis, there is a small digitiform porous appendix in the centre of the apex of the distal segment (Fig. 3). The antennae of both species seem to be morphologically indistinguishable.

L. migone - - The antenna of this species (Fig. 5) is very similar of that of $L$. longipalpis, but with a bigger proximal segment, and the distal segment smaller and narrower.

L. lenti - The antenna bears a tubercle with a small proximal segment (Fig. 6). The distal segment is ovoid. The transverse furrow of the distal segment forms a shallow invagination, showing a

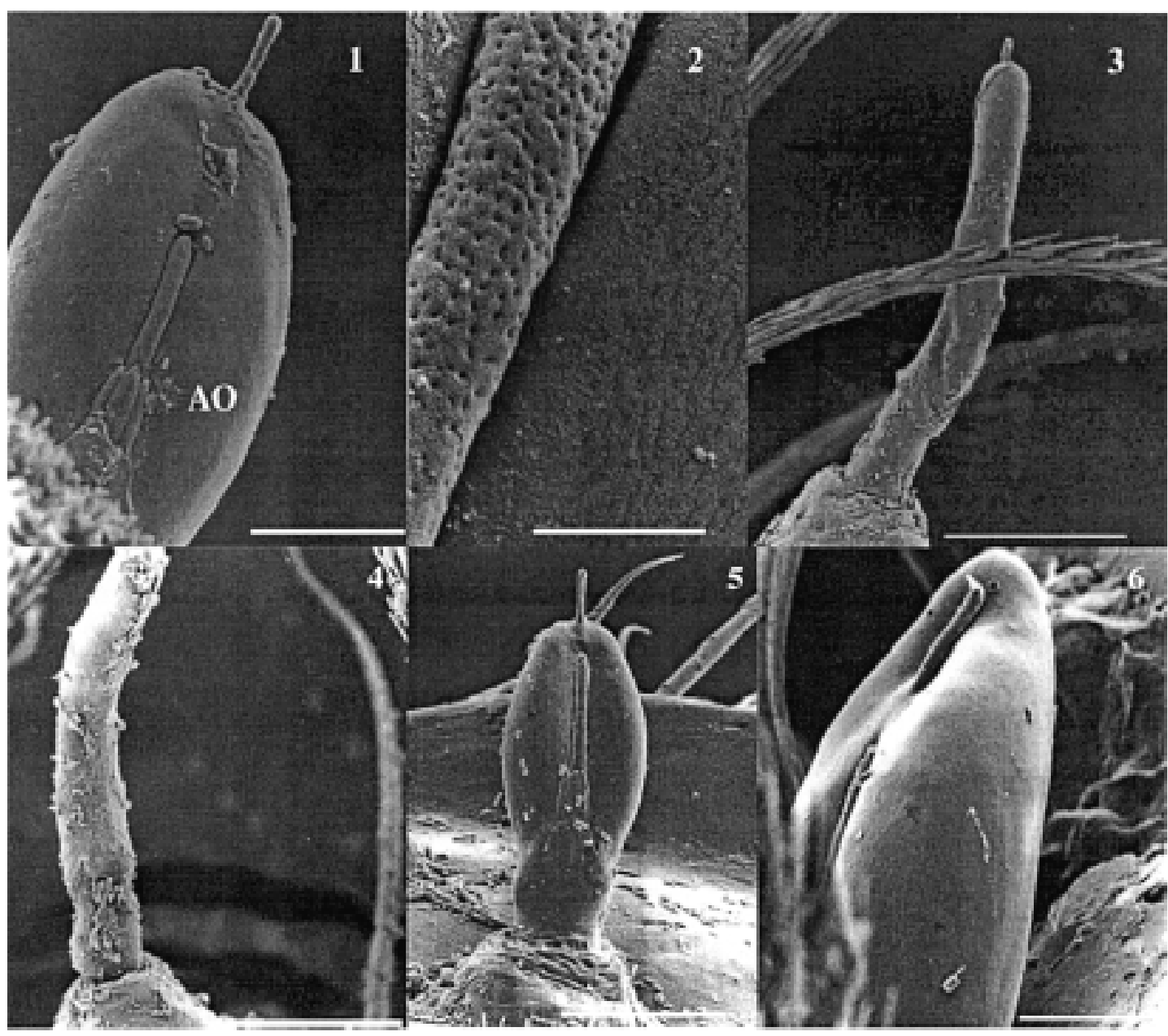

Fig. 1: Lutzomyia longipalpis antenna (bar $=9 \mu \mathrm{m}$ ). Fig. 2: detail of the L. longipalpis antenna, showing the multiporous papilla $($ bar $=1.38 \mu \mathrm{m})$. Fig. 3: L. whitmani antenna $($ bar $=22.5 \mu \mathrm{m})$. Fig. 4: L. intermedia antenna $($ bar $=20 \mu \mathrm{m})$. Fig. 5 : L. migonei antenna $(\mathrm{bar}=20 \mu \mathrm{m})$. Fig. 6: L. lenti antenna $(\mathrm{bar}=9 \mu \mathrm{m})$. AO: antenal organ 
smaller and less accentuated sensillae than the species above mentioned. In this species, the digitiform appendix, normally found in the apex in the other species, is located a little above of the centre of the antenna, inserted inside of other sensillae, showing two transversal furrows separated by a tegument layer.

$L$ sericea - The antenna is similar to that of $L$. lenti, with the distal segment presenting a ovoid format, with a transversal furrow narrower and more invaginated. The digitiform appendix is also located a little below the apex (Fig. 7). However, the antennal tubercles and the first proximal segment in this species is more prominent than in L. lenti.

L. evandroi - The antennal tubercle and the proximal segment are similar to those on the an- tenna of $L$. migonei, but the distal segment is very different from all other species examined. This segment is atrophied, the transversal furrows are not present in the centre of the antenna, and the claviform sensilla are very porous in the distal part (Fig. 8). The digitiform appendix is located in the apex of the antennae, and presents an upward curvature, of approximately 30 degrees, in the middle of the segment.

Maxillary palps

In all species examined, each maxillary palps bears maxillary organ (Fig. 9), a small circular saliency, lightly sclerotised, and are endowed with seven oniporous papiliform sensillae and three knob papillae (Fig. 10). The maxillary palps also present some trichodea setae.

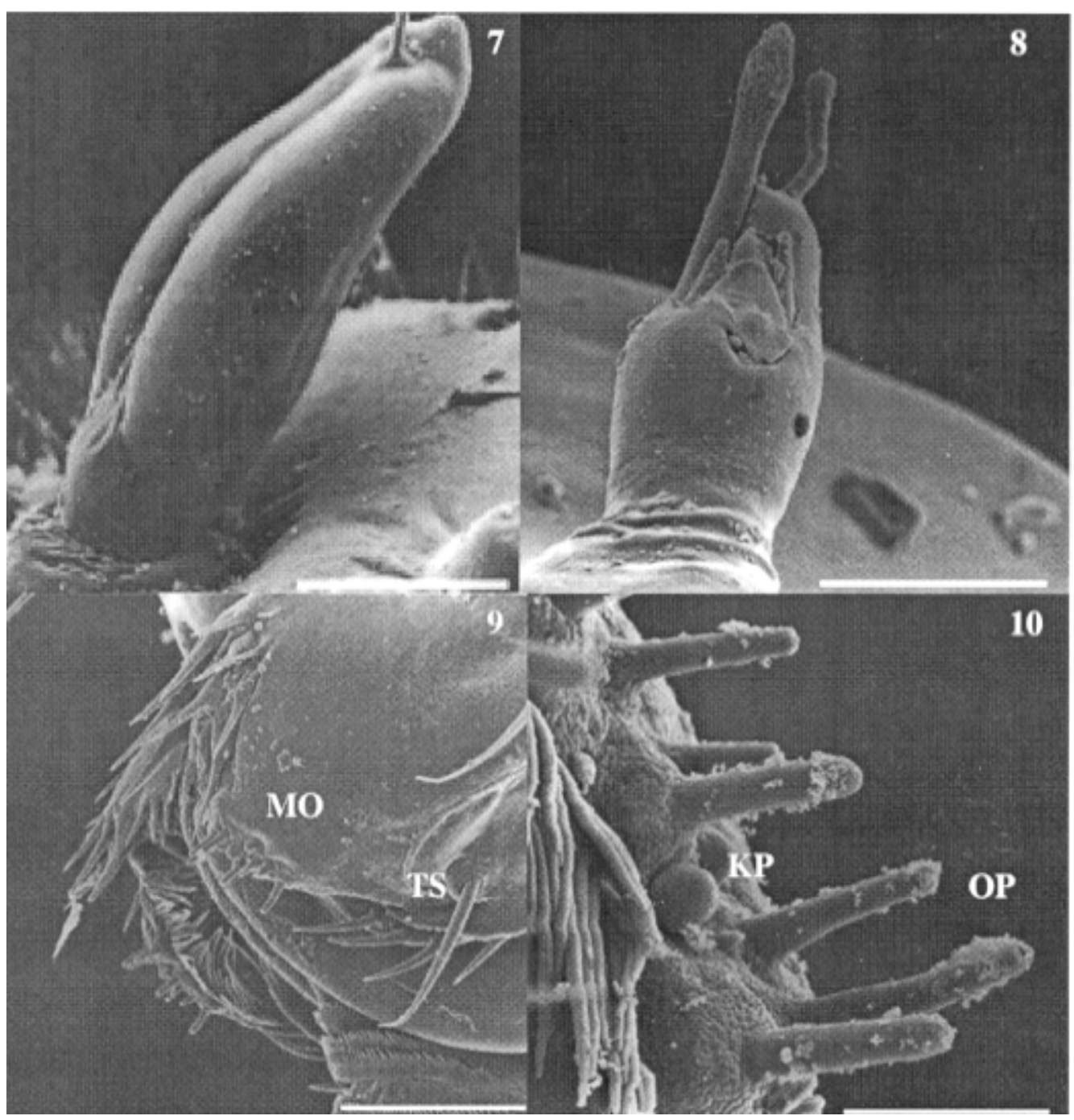

Fig. 7: Lutzomyia sericea antenna $($ bar $=13.8 \mu \mathrm{m})$. Fig. 8: L. evandroi antenna $($ bar $=9 \mu \mathrm{m})$. Fig. 9: L. migonei maxillary palp, showing the maxillary organ (MO) and trichodea setae (TS) (bar $=20 \mu \mathrm{m})$. Fig. 10: detail of the maxillary organ, showing the oniporous papillae $(\mathrm{OP})$ and the knob papillae $(\mathrm{KP})(\mathrm{bar}=4.5 \mu \mathrm{m})$ 


\section{Caudal setae}

These structures are located in the last abdominal segment of the larvae. The caudal setae present transversal furrows with a very small and scattered pores in L. longipalpis (Fig. 11), or abundant tiny pores in L. intermedia and L. whitmani (Fig. 12), $L$. lenti (Fig. 13), and L. evandroi (Fig. 14). In L. sericea (Fig. 15) the caudal setae presents espiral transversal furrows, with surrounding pores. In L. migonei (Fig. 16), the caudal setae shows transverse furrows with high numerous large pores.

\section{DISCUSSION}

The use of SEM has permitted, for the first time, the observation of the detailed morphology of the antennae, papillae of maxillary palps, and caudal setae of Lutzomyia larvae species. Sand fly larvae exhibit considerable variation in the form and position of the antennae. Hanson (1968) proposed that "in those species that burrow in their food medium in laboratory cultures (and probably in the soil in their natural habitats), the antennae are short and, in some species, somewhat very reduced in size. In surface-feeding species, however, the antennae are erect and often bear prominent tubercles". According to these approaches $L$. whitmani and $L$. intermedia must be considered surface-feeding species. The other larvae in this study may burrow in their substract.

Leite and Williams (1996) proposed four approaches for the classification of immature stages of sand flies using the morphological characters based on the form of the antenna categories: (i) antennal tubercle (socket) with the appearance of an elongate cone; basal segment short or subequal to the distal segment; (ii) antennal tubercle in the shape of the truncated cone, basal segment short, distal segment bana-shaped; (iii) antennal tubercle with the shape of a truncated cone; basal segment long or subequal the distal segment, distal segment digitiform but short; and (iv) antennal tubercle annular, basal segment short, distal segment oval.

Following these approaches, larvae of $L$. migonei, L. lenti, L. sericea and L. evandroi, all belonging to the Migonei group can be included in the last type (category iv), but some minor interspecific variations were observed among these species (Figs 5, 6, 7, 8). The antennae of $L$. longipalpis fits to category iv, although the tubercles are smaller and the distal segment stouter than those in the Migonei group. The larvae of $L$. whitmani and L. intermedia, subgenus Nyssomyia, until now, are indisguishble (Barretto 1941). No difference was observed between the antennae of these two species which fits in category iii.

The immature morphology of some families have been used for phylogenetic studies in mosquitoes (Diptera: Culicidae) (Harbach \& Peyton 1993), black flies (Diptera: Simuliidae) (Py-Daniel \& Moreira Sampaio 1994) and horseflies (Diptera: Tabanidae) (Coscarón et al. 1998). From these studies, it seems that the appearance of the phlebotomine larvae antennae might be a constant character among species of the same subgenus or spe-

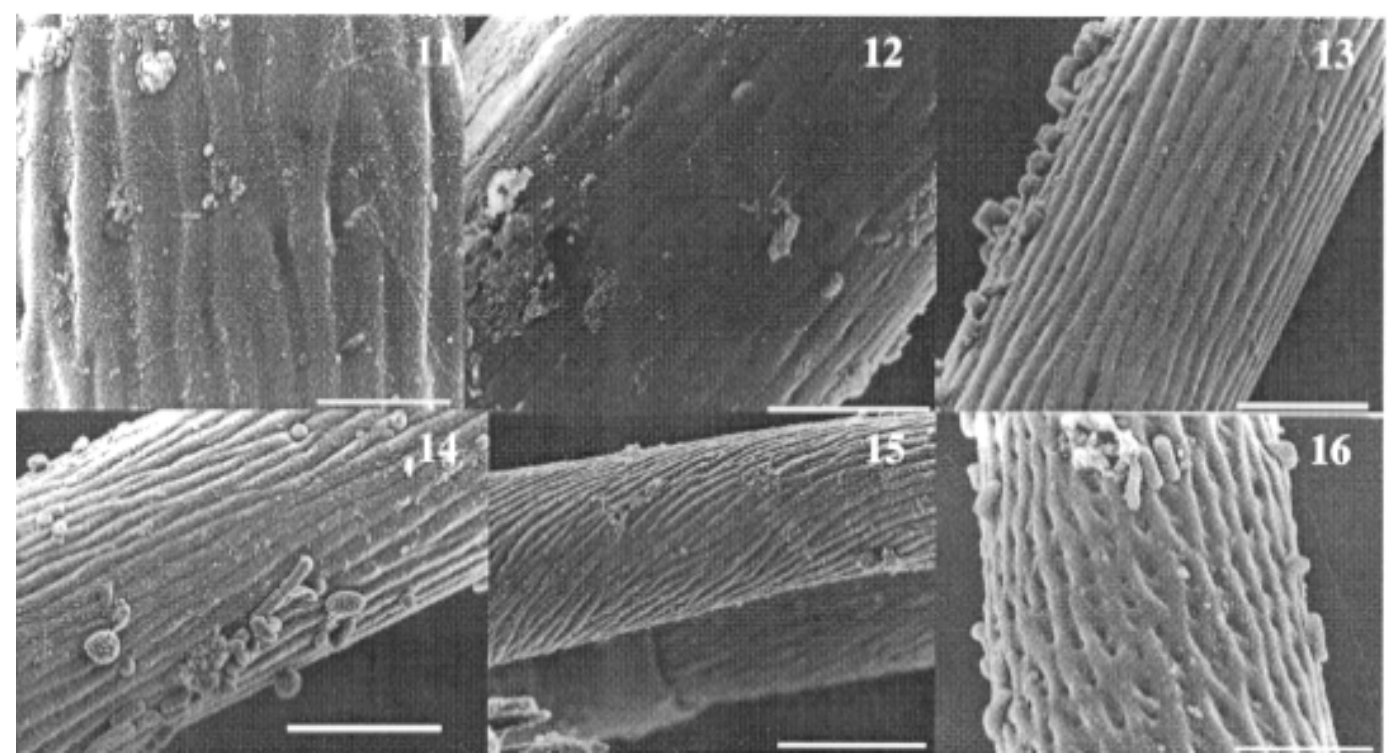

Fig. 11: Lutzomyia longipalpis caudal setae $($ bar $=1.38 \mu \mathrm{m})$. Fig. 12: L. intermedia/L. whitmani caudal setae $(\mathrm{bar}=2.5 \mu \mathrm{m})$. Fig. 13: L. lenti caudal setae $($ bar $=3.5 \mu \mathrm{m})$. Fig. 14: $L$. evandroi caudal setae $($ bar $=4.5 \mu \mathrm{m})$. Fig. 15: $L$. sericea caudal setae $($ bar $=6 \mu \mathrm{m})$. Fig. 16: L. migonei caudal setae $($ bar $=6 \mu \mathrm{m})$ 
cies group. Just as the adults, the phlebotomine larvae may have morphological features that distinguish them from another, or are subgenus-specific or group-specific. The prominent digitiform distal segment of the antenna of sand flies has so far been recorded only in larvae of species of the Nyssomyia subgenus: L. whitmani and $L$. intermedia (in present work), and $L$. umbratilis (Justiniano et al. 2001). The relevance of this feature as a distinctive immature character for Nyssomyia will only be better understood when larvae of a greater array of species of this and other subgenera or species-group are examined.

Ascoids are present on the antennae of adults phlebotomine sand flies (Forattini 1973). Dougherty et al. (1999) proved that these structures have a olfactory function. In sand fly larvae, each antennae bears two structures, similar in their morphology to the ascoids. These sstructures are multiporous suggesting a chemosensory (Zacharuk \& Shields 1991).

The papiliform structures found in the maxillary organ have a single pore in the apex of each structure (Fig. 10). According to Zacharuk and Shields (1991), oniporous papiliform structures (structures with a single pore) located in appendix of the immature mouthparts may have a gustatory function.

The caudal setae are used as the main character that distinguishes the larvae of the subfamily Phlebotominae from other subfamilies of Psychodidae (Sther 1991). According to Killick-Kendrick et al. (1989) the function of these setae is obscure, although they observed a basal sensilla on the probable points of one of the naturally absent pairs of caudal setae of $P$. tobbi. The distinctive back-andforth movement of the long setae of larvae of $L$. whitmani and L. intermedia led Barretto (1940), to suggest the presence of sensillae. In the present study, the caudal setae of all species examined bear several pores scattered along its structure, further suggesting that they may have a chemosensitive function.

Although the characters of pre-imaginal stages of insects may be as important in taxonomy as those of the adult (Emden 1957), the sand fly larvae are still too poorly known to attribute any further taxonomic significance to our observation up to the moment. Studies with TEM will be necessary to better understand the physiological function of these external structures.

\section{ACKNOWLEDGEMENTS}

To Mrs and Professor Killick-Kendrick for sand fly rearing facilities at Imperial College. To Mr Yan Whright for his invaluable the technical assistance at the SEM Laboratory, Keele University.

\section{REFERENCES}

Abonnenc E 1972. Techniques et géneralité. 3. Géneralité. In Les Plhébotomes de la Région Éthiopiennne, Mémories Orstom, Paris, p. 19-35.

Barreto MP 1941. Morfologia dos ovos, das larvas e das pupas de alguns flebótomos de São Paulo. An Fac Med S Paulo 17: 357-532.

Coscarón S, Mancebo OA, Coscarón-Arias CL 1998. The preimaginal stages of Cryptotylus unicolor (Wiedermann) and Tabanus nebulosus ornalventris Kroeber (Tabanidae - Diptera - Insecte). Mem Inst Oswaldo Cruz 93: 91-98.

Dougherty MJ, Guerin PM, Ward RG, Hamilton JGC 1999. Behavioural and electrophysiological responses of the phlebotomine sandfly Lutzomyia longipalpis (Diptera: Psychodidae) when exposed to canid host odour kairomones. Physiol Ent 24: 251-262.

Emden FI 1957. The taxomic significance of the characters of immature insects. Annu Rev Entomol 2: 91106.

Fausto AM, Feliciangeli MD, Maroli M, Mazzini M 1998. Morphological study of the larval spiracular system in eight Lutzomyia species (Diptera: Psychodidae). Mem Inst Oswaldo Cruz 93: 71-79.

Forattini OP 1973. Entomologia Médica, Vol. 4, Psychodidade; Phlebotominae; Leishmanioses; Bartoneloses, Edgard Bluncher/Edusp, São Paulo, $658 \mathrm{pp}$.

Harbach RE, Peyton EL 1993. Morphology and evolution of the larval maxila and its importance in the classification of the Sabethini (Dipetra: Culicidae). Mosq System 22: 149-159.

Hanson WS 1968. The Immature Stages of the Subfamily Phlebotomine in Panama (Diptera: Psychodidae), $\mathrm{PhD}$ Thesis, University of Kansas, Kansas, 102 pp.

Justiniano SCB, Pessoa FAC, Queiroz RG 2001. Description of the fouth-instar larva of Lutzomyia umbratilis (Diptera: Psychodidae), vector of Leishmania guyanensis (Kinetoplastida: Trypanosomatidae) in the Amazonia. Rev Soc Bras Med Trop 34 (Supl. I): 201.

Killick-Kendrick M, Killick-Kendrick R 1991. The initial establishmentnof sandfly colonies. Parassitologia 33: 315-320.

Killick-Kendrick R, Killick-Kendrick M, Leger N, Pesson B, Madulo-Leblond G, Page AM 1989. Absence of outer caudal setae on all larval instars of Phlebotomus tobii from the Ionian Greek islands. Med Vet Entomol 3: 131-135.

Leite ACR, Williams P 1996. Description of the fourth instar of Lutzomyia longipalpis, under scanning electron microscopy. Mem Inst Oswaldo Cruz 91: 571578.

Leite ACR, Williams P 1997. The first instar of Lutzomyia longipalpis, (Diptera, Phlebotomidae). Mem Inst Oswaldo Cruz 92: 197-203.

Lopes HS, Leite ACR 1987. Third contribution to the knowledge of the Raviniini (Diptera: Sarchofagidae) based on observations of the larvae, using scanning electron microscope. Mem Inst Oswaldo Cruz 82: 407-413. 
Perfil'ev PP 1968. Fauna of U.S.S.R. Diptera Phlebotomidae (Sandflies). 3. Academy of Science of the USSR, Zoological Institute New Series no. 8, p. 4659.

Pessoa FAC, Queiroz RG, Ward RD 2000. Posterior spiracles of 4th Instar larvae of four species of phlebotomine sandflies (Diptera: Psychodidae) under scanning electron microscopy. Mem Inst Oswaldo Cruz 95: 689-691.

Py-Daniel V, Moreira Sampaio RT 1994. Jalacimgomyia gen.n. (Culicomorpha); a ressurreição de Gymnopaidinae; a eliminação do nível tribal; apresentação de novos caracteres e a redescrição dos estágios larval e pupal de Simulium colombashchense (Fabrícius, 1787) (Diptera: Simuliidae). Mem CAICET 4: 101-148.

Sther FW 1991. Immature Insects, Vol 2, Knedall/Hunt Publishing Co., Iowa, 930 pp.

Ward RD 1976a. A revised numerical chaetotaxy for neotropical phlebotomine sandfly larvae (Diptera: Psychodidae). Syst Ent 1: 89-94.

Ward RD 1976b. The immature stages of some Phlebotomine sandflies from Brazil (Diptera: Psychodidae). Syst Ent 1: 227-240.

Young DG, Duncan MA 1994. Guide to identification and geographic distribution of Lutzomyia sandflies in México, the West Indies, Central and South America (Diptera:Psychodidae). Mem Amer Entomol Inst: 1-881.

Zacharuck RY, Blue SG 1971. Ultrastructure of the peg and hairsensilla on the antenna in larval of Aedes aegypti. J Morph 135: 433-456.

Zacharuck R, Shield SV 1991. Sensilla of immature insects. Annu Rev Entomol 36: 331-354.

Zacharuck RY, Ru-Siu L, Blue SG 1971. Fine structure of the antenna and its sensory cone in larvae of Aedes aegypti. J Morph 135: 273-298. 